\title{
Antibacterial and Antifungal Activity of Essential Oil of Origanum Majorana Lon a Few Bacteria and Fungi
}

\author{
Mehani $\mathrm{M}^{1 *}$, Mehani $\mathrm{I}^{2}$, Valeria $\mathrm{T}^{2}$, Morcia $\mathrm{C}^{2}$ and Segni $\mathrm{L}^{3}$ \\ ${ }^{1}$ Department of Biology, Gardaia University, Algeria \\ ${ }^{2}$ Department of Process Engineering, Faculty of Applied Sciences, Ouargla University, Algeria \\ ${ }^{3}$ CREA-GB, Research centre for Genomics and Bioinformatics, Council for Agricultural Research \\ and Economics, Italy
}

*Corresponding author: Mouna Mehani, Department of Biology, Gardaia University, Algeria,

\section{Research Article}

Volume 3 Issue 2

Received Date: November 07, 2020

Published Date: November 23, 2020

DOI: $10.23880 /$ oajmms-16000131

Email: mounameh@gmail.com

\section{Abstract}

The objective of this study is to assess the fungal and antimicrobial activity of the essential oil (ET) of marjoram (Origanum majorana L.). This strategy can contribute to the sustainable development of our country. Preliminary tests performed on the essential oil of Origanum majorana showed that this oil has antibacterial activity vis-à-vis the bacterial strains (Enterococcus feacalis, Enterobacter cloaceai, Proteus microsilis, Escherichia coli, Klebsiella pneumonia, and Pseudomonas aeruginosa) and antifungic (Fusarium sporotrichioide and Fusarium graminearum). The culture medium used was nutrient broth Muller Hinton. The interaction between the bacteria and the essential oil is expressed by a zone of inhibition with diameters of MIC indirectly expression of. And we used the PDA medium to determine the fungal activity. The extraction of the aromatic fraction (essentially oil hydrolat) of the fresh aerian part of the Origanum majorana was performed by hydrodistillation. The average essential oil yield is $0.99 \%$. The antimicrobial and fungal study of the essential oil and hydrosol showed a high inhibitory effect on the growth of pathogens.

Keywords: Essential oil; Origanum majorana; Bacteria and Fungi

\section{Introduction}

Essential oils have many therapeutic properties. In herbal medicine, they are used for their antiseptic properties against infectious diseases of fungal origin, against dermatophytes, those of bacterial origin. Humans use plants for thousands of years to treat various ailments, in many developing countries; most of the population relies on traditional doctors and their collections of medicinal plants to cure them [1].

The MAP are plants that have grown or have picks in his natural environment for its medicinal and had an infinite variety of jobs, to report the therapeutic area, food, cosmetics, industrial, etc.. Herbs can play an important role in conserving biodiversity. These plants are actually very familiar to rural people who are very sensitive to their scarcity and their disappearance. Indeed, medicinal plants play an important role of health care population and represent a significant source of income for many families in the countryside and cities [2]. Throughout history, the plant kingdom has provided the essential human resources to its feeding, hygiene and health. Since ancient times, the fragrances of these same plants are associated with mystic rites, artistic and aesthetic.

It is known that some plants emit odors to attract insects to defend themselves. These smells come from small glands on the surface of leaves, stems or flowers that contain the essential oil. The essential oil is a volatile aromatic substance extracted from the plant. Little or no fat, it is called oil because it does not mix with water. Like gasoline, it ignites. Once extracted from plants, essential oils are used in perfumery, 


\section{Open Access Journal of Mycology \& Mycological Sciences}

cosmetics, in food and other industries.

Essential oils have many therapeutic properties. In herbal medicine, they are used for their antiseptic properties against infectious diseases of fungal origin, against dermatophytes, those of bacterial origin. In this work we try to study the antibacterial and antifungal activity of essential oils on the plant: Origanum majorana.

\section{Work Methodology}

\section{Hardware Plant}

The aromatic plant harvested in the month of May 2018 is among the most abundant species in the region northwest of Algeria. The Origanum majorana: has been selected for testing antibacterial and fungal activity.

Marjoram or Garden Oregano (Origanum majorana.L) is an annual plant of the Lamiaceae family, cultivated as a condiment plant for its aromatic leaves. It is a species very close to the Common Oregano (Origanum vulgare). It is sometimes called Marjoram of the gardens. Other common names: officinal marjoram, shell marjoram [3].

Marjoram is relatively close to thyme, both in terms of its smell and its chemical composition. It belongs to the Labiaceae family. About two feet tall, marjoram is characterized by small white or purple, calyx-shaped flowers and downy, oval-shaped, gray-green leaves, growing in pairs. Wild, it is commonly known as oregano, due to its Greek etymology organon meaning "mountain and joy" or "love the mountain". It can also be called shell marjoram, from Crete, dictame, shepherd's thyme, red tea [4].

Marjoram (Origanum majorana) is an annual plant of the Lamiaceae family, cultivated as a condiment plant for its aromatic leaves. It is a species, very close to oregano, which has leaves 1 to $2 \mathrm{~cm}$ long, opposite, of a grayish green, of entire oval shape. Its flowers are small, white or mauve, arranged in tight groups in the leaf axils with two spoon-shaped bracts [5].

This herb is used in the form of fresh or dried leaves, alone or in mixture with other herbs, to flavor many culinary preparations. Marjoram is also known for its anaphrodisiac properties. It is an aromatic plant very much used in cooking, especially in Mediterranean culinary dishes, its essential oil is known for its antiseptic property [6].

\section{Classification}

Kingdom: Plantae

Subkingdom: Tracheobionta

Class: Magnoliopsida
Order: Lamiales

Family: Lamiaceae

Genus: Origanum

Species: Origanum majorana

\section{Extraction of Essential Oils by Hydrodistillation}

The hydrodistillation of Origanum majorana (leaves dry) is performed using a Clevenger-type device (1928) [2]. The setup used is shown in Figure 1.

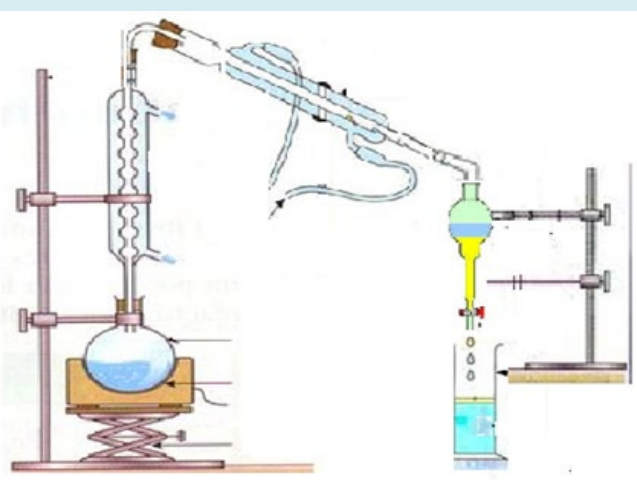

Figure 1: Mounting a Hydrodistillateur.

\section{Study of the Biological Activity of Essential Oil}

Five bacteria (Enterococcus feacalis, Escherichia coli, Enterobacter cloaceai, Klebsiella pneumoniae, Proteus microsilis and Pseudomonas aeruginosa) and two yeasts (Fusarium sporotrichioides and Fusarium graminearum) were chosen for their high frequency in human infections.

\section{Technique in Solid Medium: Method of Aromatogrammes}

The aromatogram is based on a technique used in medical bacteriology, called antibiogram $[7,8]$. It has the advantage of being very flexible in the choice of products to test and apply to many bacterial species $[9,10]$.

In this method, we use filter paper discs of $6 \mathrm{~mm}$ in diameter, impregnated in different concentrations of essential oil diluted in DMSO at 25\%, 50\% and 75\%. These discs we deposit on the surface of an agar medium inoculated with the surface of a bacterial suspension. The incubation was carried out in an oven at $35^{\circ} \mathrm{C}$ for $24 \mathrm{~h}$ for bacteria and at $25^{\circ} \mathrm{C}$ for 5 days for yeasts.

Preparing Disks: The antibiogram is starting from the preparation of records, including Whatman paper was cut into $6 \mathrm{~mm}$ white disc. After sterilizing oven pastor for $20 \mathrm{~min}$ at $160^{\circ} \mathrm{C}$, the discs were soaked in solutions with paravant prepares. Then, they are placed in Petri dishes where they 
suffered a drying before being placed on the culture medium. Preparation of Microbial Suspension: Preparation of microbial suspension is done by introducing two well isolated pure colonies of each species studied, in $10 \mathrm{ml}$ of saline contained in a test tube.

Inoculation: The microbial suspension prepared was cast on the Muller Hinton agar. After soak up of all surface of the medium by the microbial suspension, the supernatant was discarded. Therefore, these plates were left to dry for $15 \mathrm{~min}$ at $37^{\circ} \mathrm{C}$.

Application of Discs: The discs are prepared disposer to the surface of culture medium, pressing lightly with a sterile forceps, and then these dishes are incubated in an oven at $\mathrm{T}$ $37^{\circ} \mathrm{C}$ for $24 \mathrm{~h}$.

Reading Results: We have methods to measure the diameter of the zones of inhibition in the case of microbial sensitivity around the discs of $6 \mathrm{~mm}$ in diameter.

Antifungal Activity: For the realization of the antifungal activity was adopted method of direct contact. To prepare the different concentrations were taken different concentrations of essential oil of Origanum majorana namely $(50,10,5,2.5,1.25 \mathrm{mu} . \mathrm{l})$ and adjust to $20 \mathrm{ml}$ PDA then stirred for 5 minutes to homogenize the medium PDA with essential oil.

\section{Results}

\section{Antimicrobial Activity}

The study of antibacterial extracts of Origanum majorana by the agar diffusion method or the method of absorbing disc to determine the antimicrobial activity of this plant in vitro including the diameter disk $(6 \mathrm{~mm})$ to measurement of the inhibition zones.

The following Figure 2 shows the results of the antimicrobial activity of the EO (classical diffusion method) of the Origanum majorana plant on bacterial strains microsilis Proteus, Enterococcus feacalis, cloaceai Enterobacter, Klebsiella pneumoniae, Pseudomonas aeruginosa and Escherichia coli.

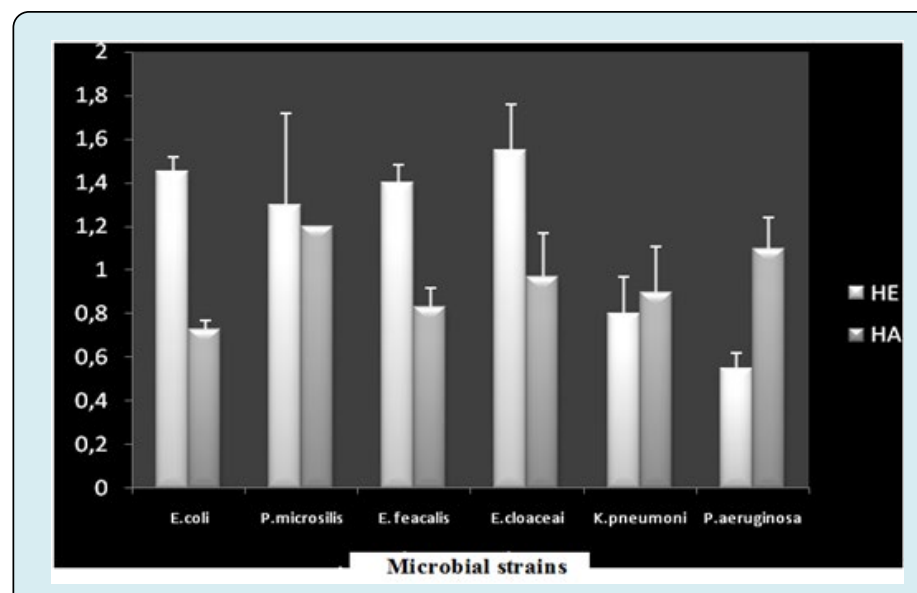

Figure 2: Antimicrobial activity of HE Origanum majorana.

The EO has in vitro inhibitory activity against the bacteria tested. By taking into consideration the inhibition diameters, EO is active on E. coli, P. microsilis, E. cloaceai and E. feacalis respectively with a muting area $1.45,1.3,1.4$ and $1.55 \mathrm{~cm}$. On the contrary, it is less active vis-à-vis P. aeruginosa and $K$. pneumoni $(0.55$ and $0.8 \mathrm{~cm})$. In contrast to $P$. aeruginosa and $P$. microsilis do not indicate great inhibitory action (insensitive) on essential oil of Origanum majorana.

Similar results were reported by [11]. They show that the essential oil of Origanum majorana Sm.a, a broad inhibitor against microorganisms studied power [12]; Also showed that the essential oils of Clausena anisata, Origanum majorana and Ocimum basilicum have biological activity on microorganisms. Our results are consistent with those reported by [13], which showed that Origanum majorana is endowed with an efficient biological activity of microorganisms.

\section{Antifungal Activity}

The antifungal activity is revealed by the absence or the presence of mycelial growth. The results of the diameter of antifungal activity of essential oil of Origanum majorana are presented in the graph 3 . They vary between 13 and $55 \mathrm{~mm}$ (diameter including the disc) in the Fusarium sporotrichioides and Fusarium graminearum, the mycelial growth is varied between 11 and $55 \mathrm{~mm}$. 


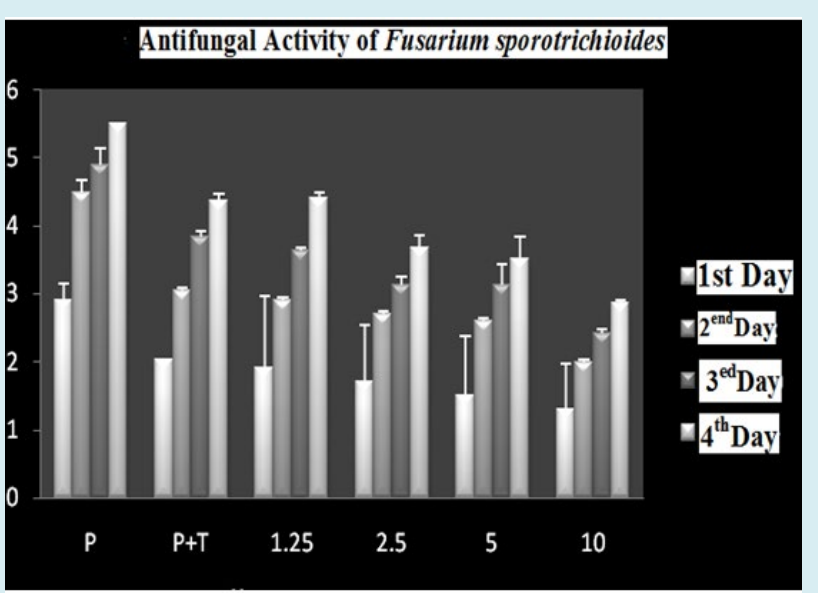

Figure 3: Antifungal Activity of Fusarium sporotrichioides.

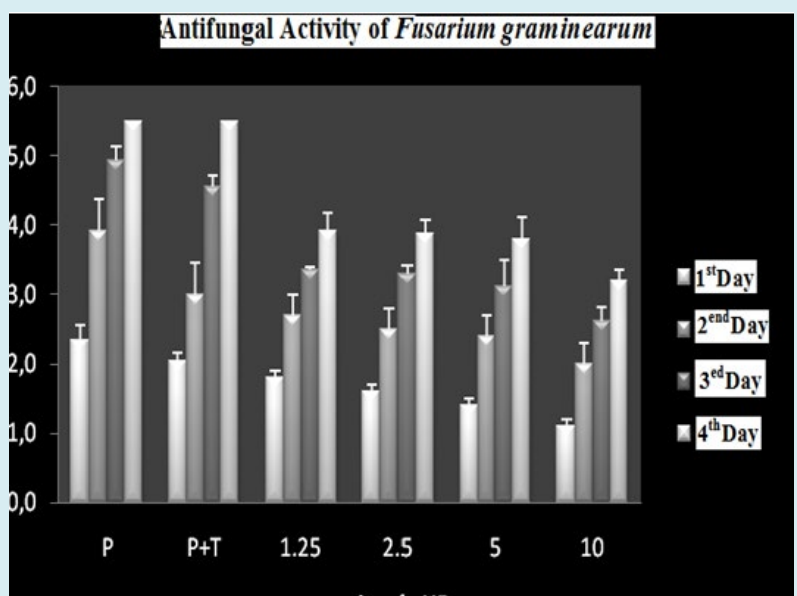

Figure 4: Antifungal Activity of Fusarium graminearum.

With different concentrations of essential oil extracted from Origanum majorana, it is observed that mycelial growth is remarkable after $72 \mathrm{~h}$ for the control and different concentrations of essential oil of Origanum majorana namely $1.25,2.5,5$ and $10 \mu \mathrm{l}$, by against at $25 \mu \mathrm{l}$ no mycelial growth of Fusarium sporotrichioides is observed.

According the graph $\mathrm{N}^{\circ} 4$, which represents the activity antifonguique of Fusarium graminearum depending on the incubation time and the concentration of essential oil of Origanum majorana we note that there is an increase in mycelial growth over time with the exception of the incubation $50 \mu \mathrm{l}$ concentration $/ 20 \mathrm{ml}$ of PDA that shows no mycelial growth.

\section{Conclusion}

Many herbs contain chemical compounds having antimicrobial properties. Several research studies have been focused on the essential oils of these herbs. The search for new therapeutic herbs character used mainly to show the validity of their use by traditional practitioners. It also showed that our country has to offer a rich and varied plant biomass. This is an immeasurable source for the development and the development of new active molecules for therapeutic purpose.

The use of volatile formulations based on medicinal and aromatic plants may have many advantages over existing products syntheses. Better understanding of our study was to Origanum majorana by studying of its aromatic fraction (HE-HA). We were able to evaluate and verify some of its biological properties and highlight its pharmacological potential.

In addition to its potential antimicrobial and antifungal verified on standardized gelose medium germs and vapor phase, the aromatic fraction has an undeniable antiinflammatoryaction. Therefore, it may be proposed eventually as an asset of choice in the local treatment of inflammation. It 


\section{Open Access Journal of Mycology \& Mycological Sciences}

might be interesting to further phytochemical and biological investigations on these plants including purification of the extracts obtained to isolate the molecules responsible for the antimicrobial activities, which will larger armamentarium of herbal plants.

\section{Acknowledgment}

Authors Could Never Accomplish This Task Without The Help of So Many Generous People. Authors would like to Acknowledge My Professor at University of Ouargla. Ladjel Segni, Prf Terzi Valeria and Morcia Caterina for their Advices, Instruction, Assistance, Patience and Many Hours at Important Edits, Re-Writes, Analysis, and Discussions; without their Example I Would not be where I am today.

\section{References}

1. Barréro F, Herrador MM, Arteaga P, Quitz J, Aksira M, et al. (2005) Chemical composition of essential oils of leaves and wood of Tetraclinis articulate (Vahl) Masters. J Essent Oil Res 17(2): 166-167.

2. Clevenger JF (1928) Determination of volatile oil. J Ann Pharm Assoc 17(4): 346-351.

3. Dubois J, Mitterand H, D Dauzat A (2006) Etymological and historical dictionary of French-Larousse.

4. Vera RR, Chane-Ming J (1999) Chemical composition of the essential oil of marjoram (Origanum majorana L.) from Reunion Island. Food Chemistry 66(2): 143-145.

5. Furia TE, Bellanca N (1971) Fenaroli's handbook of favor ingredients. The Chemical Rubber Co., Cleveland, pp: 2159.
6. Chung YK, Heo HJ, Kim EK, Kim HK, Huh TL, et al. (2001) Inhibitory effect of ursolic acid purified from Origanum majorana L. on the acetylcholinesterase. Mol Cells 11(2): 137-143.

7. Benjilali B, Tantaoui-elarki A, Ismaili-alaoui M (1986) Method for studying the antiseptic properties of essential oils by direct contact in agar medium Plant. Med Phytothé 20: 155-167.

8. Satrani B, Fougrach H, Bourkhiss B, Bousta D, Talbi M (2007) Chemical composition and antimicrobial activity of the essential oil of Cladanthus mixtus. Bull Soc Pharm Bordeaux 146: 85-96.

9. De Billerbeck VG, Roques C, Vaniere P, Marquier P (2002) Antibacterial and antifungal activity of products based on essential oils. Hygienes 10(3): 248-251.

10. Pibiri MC (2005) Microbiological sanitation of air and ventilation systems using essential oils. Doctoral thesis in science, Swiss Federal Institute of Technology Lausanne, pp: 19-55.

11. Alitonou G, Felicien Avlessi G, Valentin Wotto D, Edwige A, Dangou J, et al. (2004) Chemical composition, antimicrobial properties and activities On ticks of the essential oil of Eucalyptus tereticornis.

12. Sohounhloue DK, Agbaka A, Dockimo EB, Djossou L, Fouabi K, et al. (1999) Afr Chim 8: 87.

13. Kesbi A, Ladjel S (2011) Study of the physicochemical properties and evaluation of the biological activity of essential oils of Eucalyptus globulus in the region of Ouarglal. 